\title{
ERROR ANALYSIS OF A NEW COD CORRECTION METHOD UNITING GLOBAL AND LOCAL ORBIT FEEDBACKS
}

\author{
Y. Kamiya, N. Nakamura, M. Satoh", \\ Synchrotron Radiation Laboratory, Institute for Solid State Physics, The University of Tokyo, \\ 3-2-1 Midori-cho, Tanashi, Tokyo 188-8501, Japan
}

\begin{abstract}
A new COD correction method suitable for orbit feedback was already proposed[1]. This is the eigenvector method with constraints, which can have both functions of global and local COD corrections. In this paper, we will present the effects of errors in beam position monitor(BPM) reading and steering magnet setting on the orbit correction. We will show analytical expressions of these errors. In addition, we will show results of computer simulations for the VSX light source[2], a VUV and soft $\mathrm{X}$-ray high-brilliance synchrotron radiation source being planned at the University of Tokyo, and compare them with the analytical results.
\end{abstract}

\section{INTRODUCTION}

Photon beam positions or closed orbits in synchrotron radiation sources are usually stabilized by global and/or local feedbacks. The global feedback efficiently corrects COD around the whole ring with the harmonic method, the least squares method or the eigenvector method, while the local orbit feedback tightly fix the beam position at a photon source point by the local orbit bump method. However, the two feedbacks may interfere with each other and deteriorate the orbit stability when they are operated at the same time. We proposed a new correction method, the eigenvector method with constraints, which can have both functions of global and local COD corrections. Furthermore, the computer simulations were already carried out in only cases without any errors[1]. Since the orbit correction suffers the effect of errors in $\mathrm{BPM}$ reading and steering magnet setting, it is practical to examine the effects of such errors on COD corrections. In this paper, several such error effects are discussed and computer simulations are carried out.

\section{ERROR ANALYSIS}

\subsection{A New COD Correction Method}

In this sub-section, a new COD correction method is reviewed. The measured COD at BPMs, the kick angle strengths of the steering magnets and the response matrix are denoted by $\vec{y}, \vec{\theta}$ and $\mathrm{R}$ respectively. Here, the numbers of BPMs and steering magnets used in the correction are $\mathrm{M}$ and $\mathrm{N}$, and $\mathrm{R}$ is a MxN matrix. The residual vector of COD is defined by

$$
\vec{\Delta} \equiv \mathrm{R} \vec{\theta}+\overrightarrow{\mathrm{y}}
$$

A new COD correction method is the eigenvector method with constraints. Here, the constraint conditions are given by

$$
\overrightarrow{\mathrm{C}_{\mathrm{i}}^{\mathrm{T}}} \cdot \vec{\theta}+\mathrm{z}_{\mathrm{i}}=0\left(\mathrm{i}=1, \cdots \cdots, \mathrm{N}_{\mathrm{C}}\right)
$$

where $\mathrm{N}_{\mathrm{C}}$ means the number of constraint conditions, and the superscript " $T$ " stands for the transposed matrix.

We minimize the norm of $\vec{\Delta}$ in Eq.(1) under the constraint conditions Eq.(2) using Lagrange's method of indeterminate multipliers. After the straightforward calculations, the kick angle strengths of steering magnets using a new COD correction method are obtained by

$$
\vec{\theta}=\mathrm{By}-\mathrm{Dz}
$$

where $A=R^{T} R, P=C^{T} A^{-1} C$,

$$
\begin{aligned}
& \mathrm{B}=\left(-\mathrm{A}^{-1}+\mathrm{A}^{-1} \mathrm{CP}^{-1} \mathrm{C}^{\mathrm{T}} \mathrm{A}^{-1}\right) \mathrm{R}^{\mathrm{T}}, \\
& \mathrm{D}=\mathrm{A}^{-1} \mathrm{CP}^{-1},
\end{aligned}
$$

and the superscript "-1" stands for the inverse matrix. The inverse matrix $A^{-1}$ in Eq.(4) can be expressed by the $N_{V}$ eigenvectors and eigenvalues used for the COD correction as follows,

$$
A^{-1}=\sum_{i=1}^{N_{v}} \frac{\overrightarrow{v_{i}} \overrightarrow{v_{i}^{T}}}{\lambda_{i}},
$$

where $\overrightarrow{v_{i}}$ and $\lambda_{i}$ are the i-th eigenvector and eigenvalue of the real symmetric matrix A, respectively.

If $\mathrm{Z}_{\mathrm{i}}$ in Eq.(2) is taken as the beam position observed at an arbitrarily selected BPM and $\mathrm{C}$ as the corresponding part of the response matrix $\mathrm{R}$, the beam position can be fixed at zero.

\subsection{Errors in BPM Reading}

In this sub-section, we consider the effects of BPM errors. In order to simplify the discussion, first of all we examine separately the following 2 cases.

(i) In case of $\vec{z}=0$ and $\vec{y} \neq 0$.

\# Email: sato@issp.u-tokyo.ac.jp 
In this case, Eq.(3) is replaced by $\vec{\theta}=\overrightarrow{B y}$. Here, $\overrightarrow{\delta y}$ is BPM errors and $\delta \vec{\theta}$ is steering setting errors caused by $\delta \vec{y}$. If COD errors caused by BPM errors are denoted by $\overrightarrow{\delta \mathrm{c}}$, we can obtain,

$$
\begin{aligned}
& \delta \vec{\theta}=\mathrm{B} \delta \overrightarrow{\mathrm{y}}, \\
& \delta \overrightarrow{\mathrm{c}}=\mathrm{R} \delta \vec{\theta} .
\end{aligned}
$$

When BPM reading errors have a gaussian distribution with the standard deviation $\sigma_{\mathrm{BPM}}$, it can be expressed using Eqs.(7) and (8) as follow,

$$
\left\langle\overrightarrow{\delta c^{T}} \cdot \delta \vec{c}\right\rangle=\operatorname{Tr}\left(B^{T} R^{T} R B\right) \cdot \sigma_{\mathrm{BPM}}^{2},
$$

where the symbol "<>" and "Tr" mean the average and the trace of a matrix respectively. If the number of used eigenvalues and BPMs are denoted by $\mathrm{N}_{\mathrm{V}}$ and $\mathrm{N}_{\mathrm{BPM}}$ respectively, Eq.(9) can be represented by

$$
\left\langle\overrightarrow{\delta c^{T}} \cdot \delta \vec{c}\right\rangle=\left(N_{v}-N_{C}\right) \cdot \sigma_{B P M}^{2}
$$

or

$$
\overline{\sigma_{\mathrm{COD}}^{2}}=\frac{\mathrm{N}_{\mathrm{V}}-\mathrm{N}_{\mathrm{C}}}{\mathrm{N}_{\mathrm{BPM}}} \cdot \sigma_{\mathrm{BPM}}^{2},
$$

where the symbol " - " stands for the mean value.

Eq.(11) shows that the greater number of eigenvalues is used for correction, the $\sigma_{\mathrm{COD}}$ caused by BPM errors become worse. The factor $\left(\mathrm{N}_{\mathrm{V}}-\mathrm{N}_{\mathrm{C}}\right)$ in Eq.(11) can be interpreted as the effective number of eigenvalue used for the COD correction.

(ii) In case of $\vec{y}=0$ and $\vec{z} \neq 0$.

In this case, since Eq.(3) is represented by $\vec{\theta}=-\overrightarrow{B y}$, we can express

$$
\delta \vec{\theta}=-\mathrm{D} \delta \overrightarrow{\mathrm{z}} .
$$

If the errors of $\mathrm{z}_{\mathrm{i}}$ have a gaussian distribution with the standard deviation $\sigma_{\mathrm{z}}$, it can be expressed using Eqs.(12) and (8) as follows,

$$
\left\langle\delta \overrightarrow{c^{T}} \cdot \delta \vec{c}\right\rangle=\operatorname{Tr}\left(D^{T} R^{T} R D\right) \cdot \sigma_{z}^{2},
$$

or

$$
\overline{\sigma_{\mathrm{COD}}^{2}}=\frac{\operatorname{Tr}\left(\mathrm{P}^{-1}\right)}{\mathrm{N}_{\mathrm{BPM}}} \cdot \sigma_{\mathrm{z}}^{2} .
$$

Eq.(14) shows that the mean variance of COD varies in inverse proportion to the number of used BPMs and are proportional to the variance of $\mathrm{z}$.

In practical, $\overrightarrow{\mathrm{y}}$ and $\overrightarrow{\mathrm{z}}$ are non-zeros because these are considered as beam position at BPMs. If the correlation between beam positions at different BPMs does not exist and $\mathrm{z}$ is taken as the beam position, a variance of $\mathrm{COD}$ errors can be expressed by the summation of (i) and (ii) as follows,

$$
\overline{\sigma_{\mathrm{COD}}^{2}}=\frac{\mathrm{N}_{\mathrm{V}}-\mathrm{N}_{\mathrm{C}}+\operatorname{Tr}\left(\mathrm{P}^{-1}\right)}{\mathrm{N}_{\mathrm{BPM}}} \cdot \sigma_{\mathrm{BPM}}^{2} .
$$

\subsection{Errors in Steering Magnet Setting}

In this sub-section, we consider the effects of errors in steering magnet setting. Since the effects of errors do not depend on a COD correction scheme, the error effects in this case are completely same as that of an eigenvector method. If only the steering errors are taken into account, we can obtain

$$
\overrightarrow{\delta c^{\mathrm{T}}} \cdot \overrightarrow{\delta c}=\overrightarrow{\delta \theta^{\mathrm{T}}} \mathrm{R}^{\mathrm{T}} \mathrm{R} \delta \vec{\theta} .
$$

In the same manner as sub-section 2.2, Eq.(16) can be rewritten by

$$
\left\langle\overrightarrow{\delta \mathrm{c}^{\mathrm{T}}} \cdot \overrightarrow{\delta \mathrm{c}}\right\rangle=\operatorname{Tr}\left(\mathrm{R}^{\mathrm{T}} \mathrm{R}\right) \cdot \sigma_{\mathrm{S}}^{2}
$$

or

$$
\overline{\sigma_{\mathrm{COD}}^{2}}=\frac{1}{\mathrm{~N}_{\mathrm{BPM}}}\left(\sum_{\mathrm{i}}^{\text {all }} \lambda_{\mathrm{i}}\right) \cdot \sigma_{\mathrm{S}}^{2},
$$

where $\sigma_{\mathrm{S}}$ is the standard deviation of steering magnet steering errors, and $\sum_{i}^{\text {all }}$ means the summation of all $\lambda_{i}$, which are non-zeros.

Eq.(18) shows that the effects of errors in steering magnet setting do not depend on the number of eigenvalues used for the COD correction and the mean variance of $\mathrm{COD}$ varies in inverse proportion to the number of used BPMs.

\section{RESULTS OF SIMULATION}

The VSX project aims at constructing third-generation synchrotron light source in the Kasiwa campus of Tokyo University. The $2 \mathrm{GeV}$ VSX ring is $388 \mathrm{~m}$ in circumference. 14 insertion devices will be installed there. 128 BPMs and 112 steering magnets will be used for beam orbit feedback.

A computer simulation for the new COD correction method with BPM or steering errors has been carried out for the VSX ring. The constraints adopted here are that the positions at BPMs on the downstream sides of 14 insertion devices are zeros i.e. the number of the constraints is 14. Figure 1 shows a typical COD before correction. Here, we assumed that the alignment error of quadruple magnet has a gaussian distribution with the standard deviation $50[\mu \mathrm{m}]$. Figure 2 shows a COD after correction without any errors. Here, the symbol "»" stands for a beam position at the BPM selected as constraint and all of them are fixed at zeros.

Figure 3 and 4 show the rms ratio of CODs before and after correction with BPM reading errors and steering magnet setting errors respectively.

Figure 3 shows that the greater number of eigenvalues is used for correction, the CODs after correction become 
worse. In the sub-section 2, the correlation between beam positions at different BPMs is not taken into account. However, the result of this simulation gives good agreement with Eq.(15) as the analytical formulae qualitatively.

As shown in figure 4, the rms ratio of CODs before and after correction with steering magnet setting errors does not depend on the number of used eigenvalues for correction. This result gives good agreement with Eq.(18) as the analytical expression.

\section{CONCLUSIONS}

The effects of errors in BPM reading and steering magnet setting were analyzed, and the computer simulations were carried out using a new COD correction method. The results of these computer simulations give good agreement with the analytical expressions. According to the effects of BPM errors, a suitable number of used eigenvalues should be selected in the practical orbit feedback systems, otherwise the performance of the correction become worse.

The effects of errors in response matrix elements, the error analysis taken account of the correlation between beam positions at different BPMs and a computer simulation for the repeated correction are discussed elsewhere.

\section{REFERENCES}

[1] M. Satoh, N. Nakamura and Y. Kamiya, "A New COD Correction Method for Orbit Feedback", Proceedings of the 6th European Particle Accelerator Conference, Stockholm, p.1723, 1998.

[2] See, for instance, Y. Kamiya et al., "A Future Project of VUV and Soft X-ray High-Brilliant Light Source in Japan", Proceedings of European Particle Accelerator Conference, London, p.639, 1994.

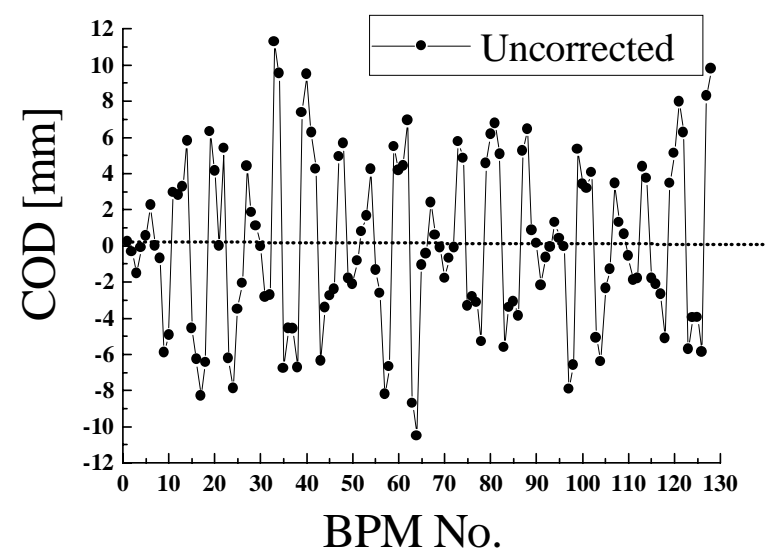

Figure 1: A typical horizontal COD without correction around the whole ring for the VSX ring.

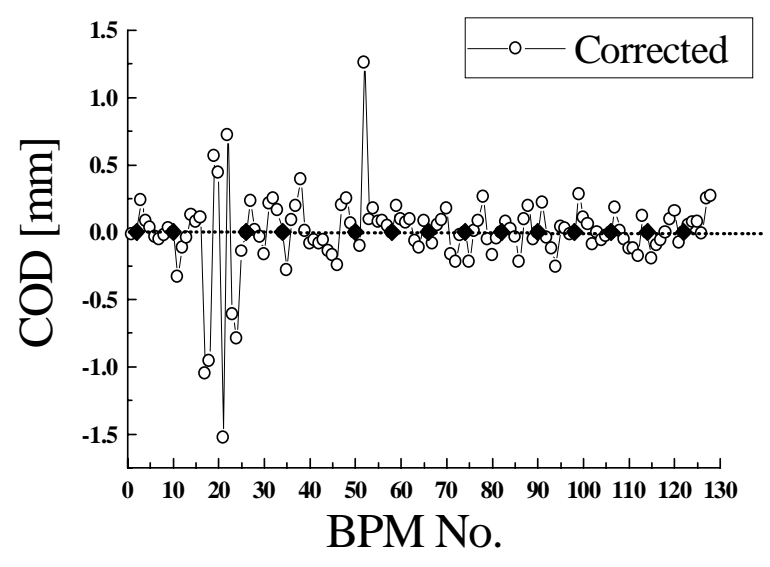

Figure 2: A horizontal COD after one turn correction using a new COD correction method.

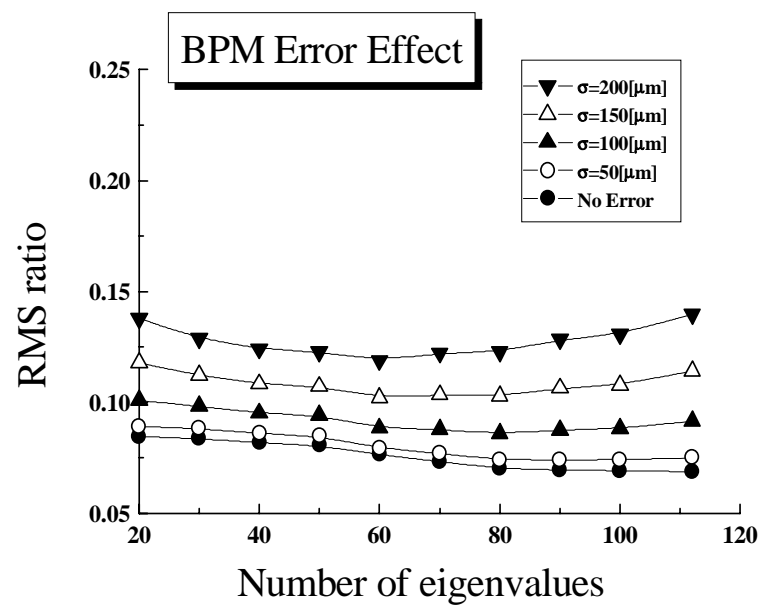

Figure 3: Rms ratio of horizontal CODs before and after correction with BPM reading errors. Here, $\sigma$ means $\sigma_{\mathrm{BPM}}$.

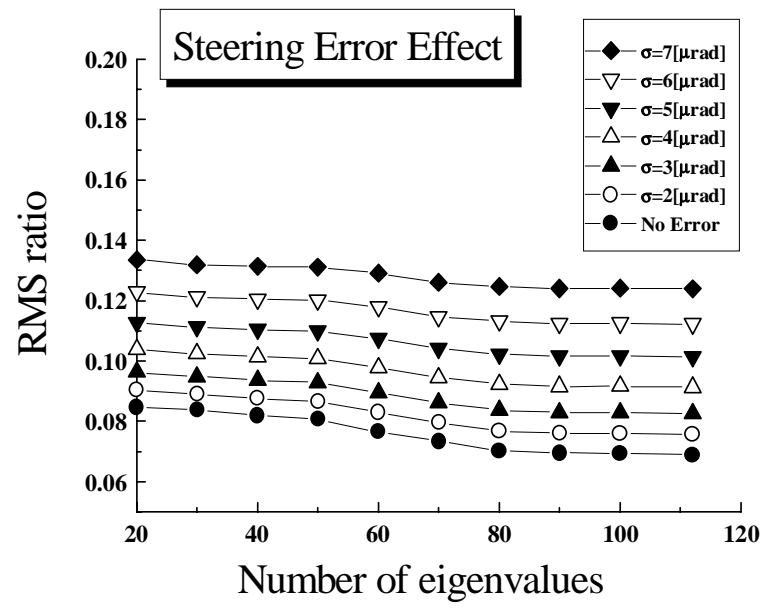

Figure 4: Rms ratio of horizontal CODs before and after correction with steering magnet setting errors. Here, $\sigma$ means $\sigma_{\mathrm{s}}$. 